\title{
TREATMENT OF RECURRENT CHRONIC BACTERIAL PROSTATITIS BY LOCAL INJECTION OF THIAMPHENICOL INTO PROSTATE
}

\author{
THEO A. PLOMP, PH.D. \\ LUC BAERT, M.D. \\ ROBERT A. MAES, PH.D. \\ From the Centre of Human Toxicology, State University of Utrecht, \\ The Netherlands, and the Department of Urology, Kliniek \\ Maria's Voorzienigheid, Kortrijk, Belgium
}

\begin{abstract}
Twenty-nine patients were treated for recurrent chronic bacterial prostatitis by an injection of $2 \mathrm{Gm}$. thiamphenicol glycinate via the perineal route directly into the prostate. Escherichia coli was identified as the pathogen responsible for this infection in 83 per cent of the cases. Using this medication locally, cure was obtained in 66 per cent of the patients. Thiamphenicol levels in prostatic fluid varied between 1 and 4,000 $\mathrm{gg} . / \mathrm{ml}$. and were unrelated to the time after intraprostatic administration. However, in most cases they were high enough to inhibit most strains of gramnegative bacilli responsible for prostatitis. Serum levels were correlated with the time after injection and decreased over twenty-four-hour observation from 25 to $0.3 \mu \mathrm{g} . / \mathrm{ml}$. The $\mathrm{pH}$ of the prostatic fluid measured in 24 patients varied from 7.1 to 8.7 with a mean value of 7.9 and was markedly higher than the $p H$ value of 6.5 reported for men without inflammatory prostatic disease. The elevated $p H$ of prostatic fluid could explain the failure of short-term trimethoprim/sulfamethoxazole (Co-trimoxazole) treatment in our patients. The cure rate of the localized thiamphenicol treatment was higher than was reported with short-and long-term trimethoprim/sulfamethoxazole therapy. We concluded that direct injection into the prostate offers a good alternative for treatment of more resistant chronic infections of the prostate.
\end{abstract}

Chronic bacterial prostatitis exists as isolated localized infections in glands of the prostate. ${ }^{1}$ It may involve only a few of many cryptic prostatic glands and varies widely in clinical manifestation. Some patients have only asymptomatic bacteriuria; most patients experience some degree of irritative voiding symptoms, such as frequency and urgency of urination, nycturia, low back pain, perineal or testicular discomfort, pain on ejaculation, and varying degrees of myalgia or arthralgia. ${ }^{1-5}$ The hallmark of chronic bacterial prostatitis is a relapsing urinary tract infection due to the same pathogen, in most cases a gram-negative coliform organism which resides in the prostatic fluid. Clinical experience has shown that a cure is difficult to achieve with most of the currently available antibacterial agents, even after prolonged usage. ${ }^{1-4}$

Experiments in the dog prostatic fistula model have demonstrated clearly that most of these agents do not diffuse from plasma into the prostatic fluid in sufficient concentrations to eradicate the infecting organism. ${ }^{6-10}$ The results of several investigations in dog and man have suggested that an antimicrobial drug effective in chronic bacterial prostatitis should have a lipid soluble base with a dissociation constant $(\mathrm{pKa})$ of 7 or greater, showing a low percentage of binding to plasma proteins, and optimal activity against gram-negative bacteria at the prostatic $\mathrm{pH}^{6,7,10-12}$ Such an antibacterial agent will diffuse readily into the prostatic fluid and even may reach a level several times higher than the 
corresponding plasma level in case the prostatic fluid is acid relative to plasma (ion-trapping phenomenon ${ }^{6}$ ).

Based on these theoretical characteristics as well as on experimental and clinical findings, a variety of antibacterial agents such as the combination of trimethoprim and sulfamethoxazole $;^{10,13,14}$ the semisynthetic tetracyclines, minocycline ${ }^{15}$ and doxycycline $;^{16,17}$ the cephalosporin antibiotic, cefazolin ${ }^{18,19}$ the macrolide antibiotics, erythromycin and rosamicin; ${ }^{20}$ the aminoglycosides, streptomycin and kanamy$\operatorname{cin} ;{ }^{12}$ and the chloramphenicol analogue, thiamphenicol, ${ }^{21}$ have been presented during the last decade as drugs which may be effective in curing infections of the prostate gland. Of these agents the combination of trimethoprim/sulfamethoxazole is currently the first-choice drug in the treatment of patients with chronic bacterial prostatitis. ${ }^{1,3-5,12}$ However, clinical experience revealed that using trimethoprim/sulfamethoxazole or one of the other antimicrobial agents, a permanent cure of prostatic infection was achieved only partly. ${ }^{1,3,4,12,21,22}$

Up to now for those patients who did not respond to antimicrobial therapy, the only alternative for cure of this disease was total or transurethral prostatectomy..$^{1-4}$ Although total prostatectomy offers a permanent cure, it often results in sexual impotence and high risk of urinary incontinence which render this solution unsatisfactory for the patient. Transurethral prostatectomy can be curative but only if all infected foci are removed successfully during surgery. Clinical experience has shown that only about one third of the patients are actually cured by this form of treatment. ${ }^{1-4}$

For these reasons it is obvious that a new mode of medical therapy which may be curative for patients with recurrent chronic bacterial prostatitis is important. In 1938 Ritter and Lippow $^{23}$ reported the use of intraprostatic injection of the antiseptic electrargol (a colloidal solution containing 0.04 per cent silver) by transurethral route as an alternative method for the treatment of this infection. Recently a new procedure based on this old technique was introduced by Baert $e t$ al..$^{24-29}$ to treat recurrent chronic bacterial prostatitis. In patients with persistent infection of the prostate after twenty days of therapy with trimethoprim/sulfamethoxazole, under rectal control they locally injected amikacin, cefazolin, or gentamicin in combination with cefazolin directly into the peripheral prostate via the perineal route.
In the present study the results of localized treatment with thiamphenicol according to this procedure are reported. Also data on the concentration of thiamphenicol in prostatic fluid and serum and on the $\mathrm{pH}$ of prostatic fluid are given. In this study thiamphenicol was chosen because of its broad-spectrum antibacterial activity resembling that of chloramphenicol, its basic character ( $\mathrm{pKa} \pm 7.2$ ), its low percentage of protein binding (10-20 per cent), its lipid solubility, its excellent diffusibility into body tissues, and its low incidence of serious side effects. ${ }^{30}$

\section{Material and Methods}

Twenty-nine patients with urine and prostatic fluid cultures positive for the same pathogen before and after treatment with 2 tablets of trimethoprim/sulfamethoxazole (Co-trimoxazole) twice a day for twenty days, were selected for injection of thiamphenicol directly into the prostate. The age of the patients varied from twenty-seven to seventy-four years (mean age, 52.5). All patients had a history of recurrent urinary tract infection, in 8 cases accompanied by orchiepididymitis. They all had been treated repeatedly with various antimicrobial agents without success.

The technique used for collecting the urine and expressed prostatic secretion specimens and for localizing the urinary tract and prostate infections has been described by Meares and Stamey. ${ }^{2,3}$ This method is based on quantitative bacterial counts of 3 separate voided urine specimens and 1 expressed prostatic secretion specimen, respectively. In our investigation, however, the second voided urine specimen $\left(\mathrm{VB}_{2}\right)$, collected before the expressed prostatic secretion specimen, was replaced by a urine specimen obtained by suprapubic puncture. ${ }^{31}$ The patients were advised to abstain from sexual intercourse for at least three days before the examination. Two to 3 drops of prostatic fluid were collected and put inmediately on dip slides by the physician during prostate massage. This procedure prevents possible contamination of the prostatic fluid specimens and guarantees that all bacteria cultured are pathogenic no matter their number.

Urine specimens were stored in plastic containers, at $4^{\circ} \mathrm{C}$. and cultured within four hours. Semiquantitative cultures were performed by surface colony counts on the dip slide culture base after twenty-four hours of incubation at $37^{\circ}$ C. Bacterial identification was done by standard methods in subcultures. 
A 20-ml. physiologic saline solution, containing $2.0 \mathrm{Gm}$. thiamphenicol glycinate (Urfamycine) and $20 \mathrm{mg}$. lignocaine hydrochloride (2 ml. Xylocaine [1\%]) was injected using a long fine needle via the perineal route directly into the prostate lobes. This procedure was performed under rectal control and without general anesthesia. During the procedure the needle was moved several times to obtain maximum distribution of the antibiotic in the prostate gland. Urine and prostatic fluid or ejaculate specimen were examined for infection at one and six months after treatment. If infection persisted after one month, localized treatment was repeated. Negative urine and prostatic fluid cultures at one and six months after treatment, respectively, indicated cure of the bacterial prostatitis.

From 33 patients, samples of serum and prostatic fluid were collected simultaneously between two and twenty-four hours after local injection of the antibiotic. In 8 cases serum and prostatic fluid samples were obtained also after the second $(n=6)$ or the third $(n=2)$ local injection into the prostate. The prostatic fluid specimens were obtained by prostatic massage and were stored at $-20^{\circ} \mathrm{C}$., together with the serum specimens, until time of assay.

To prevent urine contamination of the prostatic fluid, patients were not allowed to urinate for at least two hours prior to the prostatic massage. Thiamphenicol assays were performed using a modified ${ }^{63} \mathrm{Ni}$ electron-capture gas chromatographic method as described by Plomp, Maes, and Thiery. ${ }^{32}$ These modifications included the use of a different silylating reagent (Tri-Sil) and slightly different gas chromatographic conditions. ${ }^{21}$ The quantitation of thiamphenicol was performed by referring to a standard curve constructed from values of known amounts of thiamphenicol $(0.1-2 \mu \mathrm{g}$.) added to thiamphenicol-free samples of serum and prostatic fluid. All unknown serum $(100-500 \mu \mathrm{l}$.) and prostatic fluid $(50-100 \mu \mathrm{l}$.) samples were assayed in triplicate. In those cases in which the assayed serum and prostatic fluid concentrations were outside the range of the calibration curve, an aliquot volume of the final pyridine solution $^{32}$ was diluted after addition of internal standard solution and silylating reagent with pyridine to $1 \mathrm{ml}$.

The prostatic fluid $\mathrm{pH}$ was measured in 24 of the prostatic fluid samples, used for the thiamphenicol assay. The $\mathrm{pH}$ measurement was done using a Radiometer $\mathrm{pH}$ meter with a microelectrode at a constant temperature of $37^{\circ} \mathrm{C}$.

\section{Results}

The organisms isolated from prostatic fluid and urine specimens of 29 patients after twenty days of treatment with trimethoprim/sulfamethoxazole $(80 \mathrm{mg}$. $+400 \mathrm{mg}$.) showed the following: Escherichia coli was the most common organism (24 patients); E. coli associated with Klebsiella pneumoniae (1); Klebsiella (2); Paracoli (1); and Streptococcus faecalis ( 1 ). The other antibacterial drugs that had been used previously in these patients for the treatment of bacterial prostatitis and/or the associated recurrent urinary tract infection are listed in Table I. For recurrent urinary tract infection most patients received a course of penicillin or nitrofurantoin. Furthermore, 4 patients were not cured after oral treatment with thiamphenicol, and 8 patients did not respond to direct injections into the prostate of $4 \mathrm{Gm}$. cefazolin alone or in combination with $240 \mathrm{mg}$. of gentamicin.

The results of the localized thiamphenicol therapy in bacterial prostatitis are summarized in Table II. Nineteen of 29 patients showed negative cultures at one and six months after treatment. Of these patients, 11 were cured after one injection; among them 1 was resistant to oral thiamphenicol therapy, 5 required 2 injections, and 3 obtained sterile cultures after 3 injections. Ten of 29 patients, including 3 cases resistant to oral thiamphenicol treatment, were not cured one to two months after localized therapy. The organism recovered in all patients was similar to that identified at entry. Of these resistant cases, 5 had persistent infection even after 2 injections.

The thiamphenicol concentrations in serum and prostatic fluid at various times after injection of $2 \mathrm{Gm}$. thiamphenicol glycinate directly

TABLE I. Previous antibacterials used (all failures) in 24 patients

\begin{tabular}{lc}
\hline \multicolumn{1}{c}{ Drug } & No. of Patients \\
\hline Ampicillin & 10 \\
Nitrofurantoin & 9 \\
Gentamicin & 6 \\
Cefazolin* & 8 \\
Cefazolin* + gentamicin* & 4 \\
Thiamphenicol & 4 \\
Amoxicillin & 3 \\
Hydroxymethylnitrofurantoin & 3 \\
Doxycycline & 2 \\
Tetracycline & 1 \\
Penicillin & 1 \\
\hline
\end{tabular}

*Direct injections into the prostate. 
Figure 1. (A) Serum and (B) prostatic fluid concentrations of thiamphenicol on logarithmic scale versus time after injection of $2 \mathrm{Gm}$. thiamphenicol glycinate into prostate.
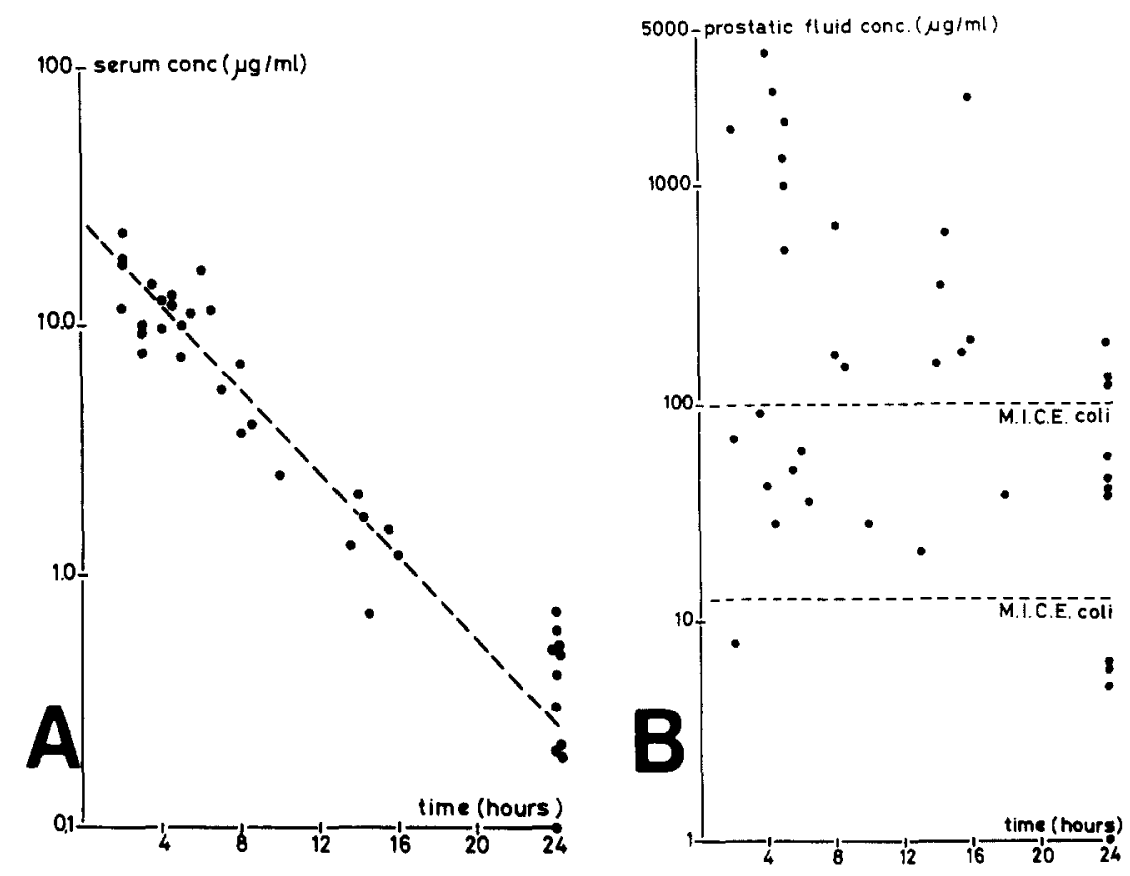

into the prostate are shown in Figure 1. The serum $(n=40)$ and prostatic fluid $(n=38)$ concentration data were obtained from the 29 studied cases and 4 additional cases with symptomatic prostatitis. The relationship of thiamphenicol serum concentration (c) versus time (t) can be expressed by the exponential function $C$ $=25.4083 \mathrm{e}^{-0.1914 \mathrm{t}}$ (Fig. 1A).

The elimination half-life $\left(t \frac{1}{2}\right)$ of thiamphenicol in serum after intraprostatic injection was 3.6 hours, and during the twenty-four hours of observation the mean thiamphenicol serum concentration decreased from 25 to $0.3 \mu \mathrm{g} . / \mathrm{ml}$. No relationship exists between the thiamphenicol prostatic fluid concentration and the time after intraprostatic injection (Fig. 1B). Large variations in the prostatic fluid concentration were found at any time during the twenty-four hours of observation, with thiamphenicol concentrations ranging from 1 to $4,000 \mu \mathrm{g} . / \mathrm{ml}$. However, irrespective of the time of administration, it can be concluded that effective thiamphenicol levels for most strains of E. coli (MIC 12.5$100 \mu \mathrm{g} . / \mathrm{ml}$.) are reached in the prostatic fluid of most patients. The $\mathrm{pH}$ values of the prostatic fluid, obtained from 24 patients with bacterial prostatitis, ranged from 7.1 to 8.7 with a mean \pm S.D. of $7.9 \pm 0.4$. In 15 of these cases the $\mathrm{pH}$ value was between 7 and 8 , and in 9 cases higher than 8 .

\section{Comment}

A cure of chronic bacterial prostatitis is difficult to achieve with most antibacterial agents because most of these compounds do not diffuse from plasma via prostatic epithelium into the prostatic fluid where the bacteria reside. ${ }^{1-4}$ Of the antibacterial drugs which are able to penetrate the prostate and kill the persistent bacteria, trimethoprim is the current agent of choice in the treatment of chronic bacterial prostatitis. ${ }^{1,3,4,5,12}$ However, clinical studies have shown that after treatment with 2 tablets of trimethoprim/sulfamethoxazole twice a day for two to four weeks only 15 to 33 per cent of the patients were cured permanently of their prostatic infection, and after treatment with the same dosage for twelve weeks a cure of 32 to 50 per cent was obtained. . $^{1,3,4,12,22,24}$

TABLE II. Results of "local" thiamphenicol therapy in bacterial prostatitis

\begin{tabular}{lcccc}
\hline \multicolumn{1}{c}{ Organisms } & $\begin{array}{c}\text { No. of } \\
\text { Patients }\end{array}$ & $\begin{array}{c}\text { Neg. } \\
\text { Culture* }\end{array}$ & Relapset & $\begin{array}{c}\text { Recurrent } \\
\text { Organism }\end{array}$ \\
\hline E. coli & 24 & 16 & 8 & E. coli \\
E. coli/Klebsiella & 1 & 1 & 0 & $\ldots$ \\
Klebsiella & 2 & 2 & 0 & $\ldots$ \\
Paracoli & 1 & 0 & 1 & Paracoli \\
Streptococcus & 1 & 0 & 1 & Streptococcus \\
\hline
\end{tabular}

*Negative urine and prostatic fluid culture at one and six months after last treatment.

$\nmid$ Positive urine and prostatic fluid culture between one and two months after last treatment. 
Up to the present time in those patients not responding to the aforementioned therapy, a cure could have been obtained by oral or parenteral treatment with other possibly effective agents such as minocycline, doxycycline, cefazolin, streptomycin, kanamycin, and thiamphenicol $^{12,15-17,21}$ or by removal of the infected foci by transurethral resection. ${ }^{1-4}$ We present a new alternative medical treatment of chronic bacterial prostatitis involving direct injections of thiamphenicol into the prostate. This therapy proved to be effective in controlling prostatic infections in 19 ( 66 per cent) of 29 patients (Table II).

To evaluate the cure rate the patients were divided into two groups according to medication used previously. Group 1, 17 patients, had been treated previously with urinary tract disinfectants nitrofurantoin or ampicillin and a standard three-week therapy of trimethoprim/ sulfamethoxazole. Group 2, 12 patients, had received besides the aforementioned treatment local injections of cefazolin alone or in combination with gentamicin, and/or intramuscular injection of gentamicin, or an oral course of thiamphenicol or doxycycline (Table I).

In these 2 groups the cure rates were 71 and 58 per cent, respectively, and they indicate that localized treatment with thiamphenicol is about as effective in the uncomplicated as in the more resistant cases of chronic bacterial prostatitis. Compared to the reported cure rates after shortand long-term therapy with trimethoprim/ sulfamethoxazole, ${ }^{1,3,4,12,22,24}$ our cure rate may be considered to be favorable. Further, the cure rate after local injection of thiamphenicol was comparable to that obtained in 10 patients with chronic bacterial prostatitis after oral treatment (500 mg. of thiamphenicol, 3 times a day for six weeks). ${ }^{21}$ It should be noted that in the latter case, first, a smaller number of patients had been treated, and second the patients received only trimethoprim/sulfamethoxazole before the thiamphenicol therapy was initiated and therefore should be regarded as uncomplicated cases.

Concerning the organisms causing bacterial prostatitis, our study clearly indicates that gram-negative organisms are responsible primarily for this infection. In 83 per cent of our cases the infection was due to various strains of $\mathrm{E}$. coli and to Klebsiella, Paracoli, S. faecalis, and a mixed infection of $\mathrm{E}$. coli/Klebsiella in the remaining 17 per cent. These findings are in agreement with the results reported by Meares and Stamey, ${ }^{2}$ implicating E. coli as the most common pathogen in chronic bacterial prostatitis. In our study prostatic fluid levels indicate that no relationship exists between the thiamphenicol concentration in prostatic fluid and the time after injection into the prostate.

The high variations in prostatic fluid concentrations at any time may be explained by the fact that in each case of chronic bacterial prostatitis different degrees of inflammation of the prostatic tissue are likely to be encountered, resulting in variable diffusion into the prostatic fluid. Also in each patient complete application of the antibiotic only in prostatic tissue is not always feasible, consequently resulting in lower attainable levels in prostatic fluid. The minimum inhibitory concentration (MIC) of thiamphenicol for most strains of $\mathrm{E}$. coli ranges between 12.5 and $100 \mu \mathrm{g} . / \mathrm{ml} .{ }^{30}$ Comparing these MIC values to the observed prostatic fluid levels (Fig. 1B), it may be concluded that after local injection effective levels are reached in most cases.

Data on the prostatic fluid level after intravenous (IV) administration of $2 \mathrm{Gm}$. thiamphenicol glycinate are not available. However, a mean ejaculate thiamphenicol concentration of $2.3 \mu \mathrm{g} . / \mathrm{ml}$. at two hours after a single oral administration of $500 \mathrm{mg}$. of the drug and a mean prostatic tissue level of $32 \mu \mathrm{g}$. $/ \mathrm{Gm}$. at forty-five minutes and of $6 \mu \mathrm{g} . / \mathrm{Gm}$. at two hours, respectively, after injection of $1 \mathrm{Gm}$. were reported previously. ${ }^{21,33}$ From these data a prostatic fluid concentration of about $10-20 \mu \mathrm{g} . / \mathrm{ml}$. at two hours after IV injection of $2 \mathrm{Gm}$. of the antibiotic can be expected, which in most cases is considerably lower than the levels obtained after local injection of the same dose (Fig. 1B).

After intraprostatic injection of $2 \mathrm{Gm}$. of thiamphenicol glycinate the mean serum level decreased from $17 \mu \mathrm{g} . / \mathrm{ml}$. at two hours to 0.3 $\mu \mathrm{g} . / \mathrm{ml}$. at twenty-four hours (Fig. 1A). Intravenous administration of the same dose to adults with normal renal function resulted in comparable serum values at the same times. On these grounds it may be suggested that after injection into the prostate a rapid diffusion from prostatic tissue to blood takes place. ${ }^{25}$ In our cases of chronic bacterial prostatitis a mean $\mathrm{pH}$ of 7.9 of the prostate fluid was observed. This value was in agreement with the mean $\mathrm{pH}$ of 7.7 and 8.1 in patients with chronic bacterial prostatitis reported by Blacklock and Beavis ${ }^{11}$ and Pfau and Sacks, ${ }^{12}$ respectively. Comparing these mean $\mathrm{pH}$ values with the prostatic fluid pH of 6.5 found in dogs and normal men, it may be concluded that the presence of infection and 
inflammation of the prostate causes a significant elevation of the prostatic fluid $\mathrm{pH}$ and in turn a high alkaline $\mathrm{pH}$ may serve as an indication of a prostatic infection. ${ }^{6,7}$

In addition the elevated prostatic fluid $\mathrm{pH}$ may be used to explain failure of short-term trimethoprim/sulfamethoxazole treatment to cure chronic bacterial prostatitis. By elevation of the prostatic $\mathrm{pH}$ as observed in our patients, the previous $\mathrm{pH}$ gradient between the plasma and the prostatic fluid is abolished or even may be reversed. In such a case a lipid soluble base with a pKa of 7.4, like trimethoprim, no longer will be able to concentrate adequately in the prostate (ion-trapping phenomenon ${ }^{6}$ ) and even may reach a higher concentration in the plasma. Consequently no cure will be obtained since the attained trimethoprim levels then will be insufficient for the eradication of gram-negative bacteria in the prostatic fluid.

Finally, in our opinion, the new localized thiamphenicol treatment has several advantages over the currently most used long-term trimethoprim/sulfamethoxazole treatment: direct presence of highly active antibiotic concentration at the site of infection, minimal incidence of side-effects, no limitation of concentration in prostatic fluid of high alkaline $\mathrm{pH}$, and no possible inactivation of the antibiotic by metabolism in the body. The pain and discomfort experienced by the patients during direct injection into the prostate were minimal and were comparable to that observed after intramuscular injection.

The data of the current study have demonstrated that thiamphenicol enters the prostatic fluid in therapeutic levels after direct injections into the prostate and is curative in 66 per cent of the cases of resistant chronic bacterial prostatitis: Therefore we recommend this as treatment of choice for chronic bacterial prostatitis, especially for the more resistant cases. The effectiveness of local thiamphenicol injections in cases that are resistant to oral therapy with the drug is questionable, and in these cases local treatment with other antibiotics is advisable.

3521 GE Utrecht, The Netherlands (DR. PLOMP)

ACKnowledgment. The technical expertise of Mrs. W. C. van Ditmarsch.

\section{References}

1. Drach GW: Prostatitis: man's hidden infection, Urol. Clin. North Am. 2: 499 (1975).

2. Meares EM Jr, and Staney TA: The diagnosis and manage- ment of bacterial prostatilis, Br. J. Urol. 44: 175 (1972).

3. Meares EM Jr: Prostatitis, a review, Urol. Clin. North Am. 2: 3 (1975).

4. IDEM: Prostatitis: diagnosis and treatment, Drugs 15: 472 (1978).

5. Berkow R: The Merck Manual of Diagnosis and Therapy, 13th ed., Rahway, N.J., Merck Co., Inc., 1977, p. 719.

6. Stamey TA, Meares EM Jr, and Winningham DG: Chronic bacterial prustatitis and the diffusion of drugs into prostatic fluid, J. Urol. 103: 187 (1970).

7. Winningham DG, and Stamey TA: Diffusion of antibiotics from plasma into prostatic fluid, Nature 219: 139 (1968).

8. IDEM: Diffusion of sulfonamides from plasma into prostatic fluid, J. Urol. 104: 5.59 (1970).

9. Hessl JM, and Stamey TA: The passage of tetracyclines across epithelial membranes with special reference to prostatic epithelium, ibid. 106: 253 (1971).

10. Reeves DS, and Ghilchick M: Secretion of the antibacterial substance trimethoprim in the prostatic fluid of dogs, Br. J. Urol. 42: 66 (1970).

11. Blacklock NJ, and Beavis JP: The response of prostatic fluid $\mathrm{pH}$ in inflammation, ihid. 46: 537 (1974).

12. Pfau $A$, and Sacks $T$ : Chronic bacterial prostatitis: new therapeutic aspects, ibid. 48: 245 (1976).

13. Oosterlinck W, Defoort R, and Renders G: The concentration of sulphamethoxazole and trimethoprim in human prostate gland, ibid. 47: 301 (1975).

14. Dabhoiwala NF, Bye $A$, and Clardige $M$ : A study of concentrations of trimethoprim-sulphamethoxazole in the human prostate gland, ibid. 48: 77 (1976).

15. Hensle TW, Prout GR, and Griffin D: Minocycline diffusion into benign prostatic hyperplasia, J. Urol. 118: 609 (1977).

16. Fabre J, et al: La cinctique des tetracyclines chez l'homme, Schweiz. med. Wschr. 101: 625 (1971).

17. Garnes HA: Doxycycline levels in serum and prostatic tissue in man, Urology 1: 205 (1973).

18. Litvak AS, et al: Cefazolin and cephalexin levels in prostatic tissue and sera, ibid. 7: 497 (1976).

19. Suzuki $\mathrm{K}$, et al: Diffusion of antibacterial drugs into human prostatic fluids, Proceedings of the 10th International Congress of Chemotherapy, Zürich, 1978, p. 412.

20. Baumueller A, Hoyme U, and Madsen PO: Rosamicin, a new drug for the treatment of bacterial prostatitis, Antimicrob. Agents Chemother. 12: 240 (1977).

21. Plomp TA, Mattelaer JJ, and Maes RAA: The concentration of thiamphenicol in ejaculate and prostatic tissue, J. Antimicrob. Chemotherap. 4: 65 (1978)

22. Paulson DF, and Devere White R: Trimethoprimsulfamethoxazole and minocycline hydrochloride in the treatment of culture-proved bacterial prostatitis, J. Urol. 120: 184 (1978).

23. Ritter SJ, and Lippow C: Pathological and bacteriological processes present in prostatitis and tissue reaction to therapy, ibid. 39: 111 (1938).

24. Baert L, Soep H, and Pyck J: Chronische bacteriële Prostatitis, Tschr. geneesk. 16: 794 (1975).

25. IDEM: Chronic bacterial prostatitis, Ann. Urol, 10: 39 (1976)

26. Baert L: Bacterial prostatitis treated by local antibiotics, letter to the editor, Urology 8: 644 (1976).

27. IDEM: Bacterial prostatitis and its present treatment, Ann. Urol. 11: 275 (1977).

28. IDEM: Direct antibiotic injection clears most prostatitis, Family Prac. News 7: 57 (1977).

29. IDEM: A reappraisal of treatment in chronic bacterial prostatitis, letter to the editor, J. Urol, in press.

30. Farrari V: Thiamphenicol, experimental and clinical basis of a new antibiotic, Zambon S.p.A., Milan, 1968.

31. Baert L: Urine analysis, estimation of residue and cystography using the suprapubic approach, Ann. Urol. 7: 83 (1973)

32. Plomp TA, Maes RAA, and Thiery M: A rapid-gas chromatographic determination of thiamphenicol in plasma and amniotic fluid, J. Chromatogr. 121: 243 (1976).

33. Plomp TA, Schalkhäuser KM, and Maes RAA: Concentration of thiamphenicol in the human prostate and testis, Chemotherapy 25: 254 (1979). 